\title{
Atracurium and vecuronium interaction with gentamicin and tobramycin
}

J.Y. Dupuis MD, R. Martin MD FRCP, J.-P. Tétrault MD MSc FRCP
Drug interactions between aminoglycoside antibiotics and neuromuscular blocking agents are well known.' Many in vitro and in vivo animal studies have shown that aminoglycoside antibiotics potentiate the action of nondepolarizing muscle relaxants. These findings have been correlated in humans by many case reports where aminoglycoside antibiotics either increased the duration of action of muscle relaxants, or caused recurrence of the neuromuscular blockade produced by d-tubocurarine, pancuronium or vecuronium ${ }^{2}$ after reversal of the block. Except for those case reports, there are no clinical trials in man on the effects of aminoglycosides on neuromuscular block produced by muscle relaxants.

The present study was undertaken to determine the effects of gentamicin and tobramycin, when used at therapeutic serum levels, on the neuromuscular block produced by vecuronium and atracurium in man.

\section{Methods}

The study was approved by the Hospital Ethics Committee and informed written consent was obtained from all patients. Forty-four adult patients of both sexes, ASA physical status I to III, without neuromuscular disease or other conditions affecting muscle relaxants, were included in the prospective study. Various types of surgery were performed, but they were mainly orthopaedic and urological procedures, hysterectomies or inguinal herniorraphies.

The patients were divided into four groups. Group 1 consisted of 14 patients already treated with gentamicin or tobramycin who received atracurium $0.5 \mathrm{mg} \cdot \mathrm{kg}^{-1}$. They were compared with 14 control patients not receiving antibiotics (Group 2). Group 3 consisted of eight patients treated with gentamicin or tobramycin who received vecuronium $0.1 \mathrm{mg} \cdot \mathrm{kg}^{-1}$. They were compared with eight control patients not receiving antibiotics (Group 4). At the time of induction of anaesthesia, all patients in groups 1 and 3 has a blood sample taken to measure the serum level of gentamicin or tobramycin.

All patients received diazepam $0.15 \mathrm{mg} \cdot \mathrm{kg}^{-1} \mathrm{PO}$ as premedication and a similar anaesthetic technique was used in all 44 patients. General anaesthesia was induced 

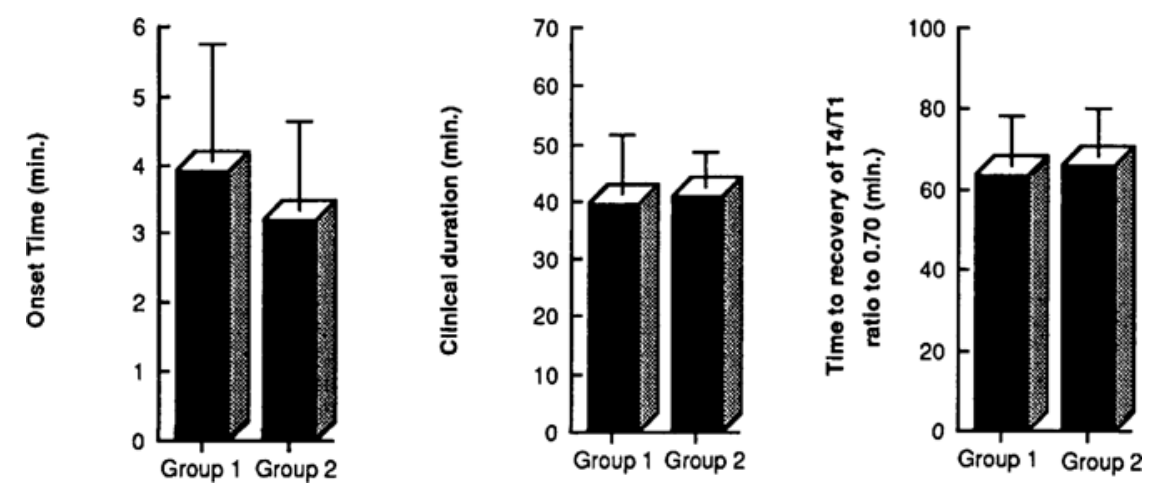

FIGURE I Atracurium-gentamicin or tobramycin (Group I) and atracurium-controls (Group 2) with regard to onset time, clinical duration and time to spontaneous recovery of $\mathrm{T} 4 / \mathrm{T} 1$ ratio of 0.70 (minutes \pm SD) after administration of atracurium.

with sufentanil $1 \mathrm{\mu g} \cdot \mathrm{kg}^{-1}$ and thiopentone 2 to 6 $\mathrm{mg} \cdot \mathrm{kg}^{-1}$. Muscle relaxation was then induced with an initial bolus dose of atracurium $0.5 \mathrm{mg} \cdot \mathrm{kg}^{-1}$ for patients in Groups 1 and 2 , and vecuronium $0.1 \mathrm{mg} \cdot \mathrm{kg}^{-1}$ for patients in Groups 3 and 4 . Anaesthesia was maintained by inhalation of isoflurane to a maximum of 0.25 to 0.5 per cent (inspired) in a mixture of nitrous oxide 70 per cent and oxygen 30 per cent. Sufentanil supplementation 0.5 to $1 \mu \mathrm{g} \cdot \mathrm{kg}^{-1} \cdot \mathrm{h}^{-1}$ of surgery was administered, as needed. Additional injections of muscle relaxants were administered if necessary, but only after spontaneous recovery of a $\mathrm{T} 4 \mathrm{~T} 1 \mathrm{latio}$ of 0.70 following the initial dose of atracurium or vecuronium. At the end of the procedure, the neuromuscular blockade was antagonized with neostigmine $0.04 \mathrm{mg} \cdot \mathrm{kg}^{-1}$ and glycopyrrolate $0.015 \mathrm{mg}$. $\mathrm{kg}^{-1}$.

Neuromuscular function was monitored by electromyography (EMG) with a Datex NMT 221 monitor (PuritanBennett, Helsinki, Finland). The electromyographic response of the hypothenar muscles was measured after supramaximal train-of-four (TOF) stimuli, at a frequency of $2 \mathrm{~Hz}$, delivered to the ulnar nerve every 20 seconds. After the initial dose of muscle relaxants, the following variables were assessed: onset time, defined as the time between the administration of muscle relaxant and 90 per cent twitch depression; clinical duration, defined as the time between administration of muscle relaxant and 25 per cent twitch recovery; and time to spontaneous recovery of $\mathrm{T} 4 / \mathrm{T} 1$ ratio of 0.70 after administration of the muscle relaxant.

In order to detect any recurrence of neuromuscular blockade one hour after antagonism with the anticholinesterase, the neuromuscular function of the patients was reassessed by one of two methods: awake patients were asked to sustain a head lift for five seconds; sleeping patients received a $50 \mathrm{~Hz}$ supramaximal tetanus at the ulnar nerve, for five seconds, with the muscular response of the adductor pollicis being assessed by a tactile method.

An unpaired Student's $t$ test was used to compare Group 1 with Group 2, and Group 3 with Group 4, for age, weight, onset time, clinical duration and time to spontaneous recovery of T4/T1 of 0.70 . The Fisher exact probability test was used to compare sex distribution. $\mathrm{P}<0.05$ was considered significant. Simple linear regression was also used to evaluate the association between aminoglycoside serum levels and the clinical duration of the muscle relaxants, and the time to spontaneous recovery of T4/T1 ratio of 0.70 after the administration of the muscle relaxants.

\section{Results}

No significant differences were found for age, weight and sex distribution, between the patients in Group 1 (aminoglycosides) and those in Group 2 (control) who received atracurium, or between the patients in Group 3 (aminoglycosides) and those in Group 4 (control) who received vecuronium (Table).

Considering onset time, clinical duration, and time to spontaneous recovery of a $\mathrm{T} 4 / \mathrm{T} 1$ ratio of 0.70 , no statistically significant difference could be observed between Group 1 (aminoglycoside-atracurium) and Group 2 (control-atracurium) (Figure 1). However, statistically significant differences were observed between Group 3 (aminoglycoside-vecuronium) and Group 4 


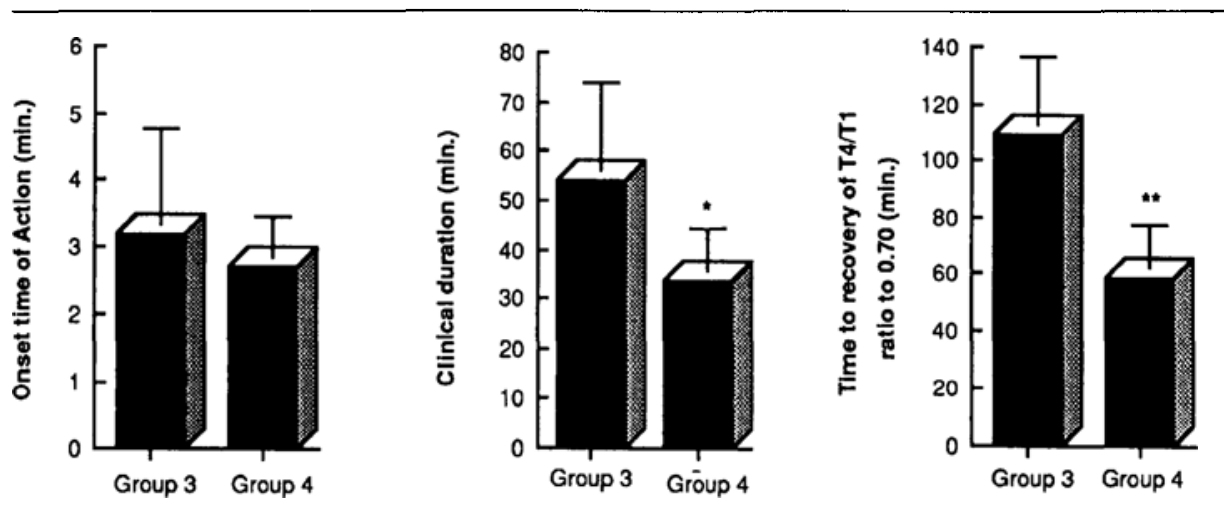

FIGURE 2 Vecuronium-gentamicin or tobramycin (Group 3) and vecuronium-controls (Group 4) with regard to onset time, clinical duration and time to spontaneous recovery of $T 4 / T 1$ ratio of 0.70 (minutes $\pm S D$ ) after administration of vecuronium. $* P<0.01, * * P<0.0005$.

(control-vecuronium) with clinical duration $(\mathbf{P}<0.01)$ and with time to spontaneous recovery of a $\mathrm{T} 4 / \mathrm{T} 1$ of 0.70 $(\mathrm{P}<0.0005)$, those two variables being longer in Group 3 than in Group 4 (Figure 2).

All the patients receiving aminoglycoside antibiotics had serum concentrations in the therapeutic range: $5 \pm$ $2.4 \mu \mathrm{g} \cdot \mathrm{ml}^{-1}$ (mean $\pm \mathrm{SD}$ ) in the atracurium group (Group 1) and $3.7 \pm 1.9 \mu \mathrm{g} \cdot \mathrm{ml}^{-1}$ in the vecuronium group (Group 3). No significant difference was found between these values.

Eight patients were treated with tobramycin and 14 with gentamicin. No significant correlation between the antibiotic serum concentrations and the clinical duration or the time to spontaneous recovery of a $\mathrm{T} 4 / \mathrm{T} 1$ ratio of 0.70 after administration of the muscle relaxants was observed in Group 1 or in Group 3. Patients taking tobramycin were compared with those taking gentamicin in Group 3. No significant differences could be demonstrated between the two subgroups for the measured

TABLE Age, weight and sex distribution of the patients (mean \pm SD)

\begin{tabular}{|c|c|c|c|c|}
\hline & \multicolumn{2}{|c|}{ Atracurium $0.5 \mathrm{mg} / \mathrm{kg}^{*}$} & \multicolumn{2}{|c|}{ Vecuronium $0.1 \mathrm{mg} / \mathrm{kg}^{\dagger}$} \\
\hline & $\begin{array}{l}\text { Group I } \\
\text { amiglycoside } \\
(n=14)\end{array}$ & $\begin{array}{l}\text { Group } 2 \\
\text { control } \\
(n=14)\end{array}$ & $\begin{array}{l}\text { Group } 3 \\
\text { aminoglycoside } \\
(n=8)\end{array}$ & $\begin{array}{l}\text { Group } 4 \\
\text { control } \\
(n=8)\end{array}$ \\
\hline \multicolumn{5}{|l|}{ Age } \\
\hline (years) & $57.4 \pm 19$ & $52.1 \pm 17.3$ & $42 \pm 13$ & $45 \pm 17$ \\
\hline Weight & $66.3 \pm 11.8$ & $69.0 \pm 20.5$ & $71.2 \pm 7.8$ & $725 \pm 10.9$ \\
\hline \multicolumn{5}{|l|}{ Male } \\
\hline female & $7 / 7$ & $6 / 8$ & $7 / 1$ & $4 / 4$ \\
\hline
\end{tabular}

*G1 vs G2: $P=$ N.S. (age, weight, sex ratio)

tG3 vs $G 4: P=$ N.S, (age and weight, sex ratio) variables (clinical duration and time to spontaneous recovery of $\mathrm{T} 4 \mathrm{~T} 1$ ratio of 0.70 ).

All patients, except one in Group 3 (tobramycinvecuronium) were either able to maintain their head lift for five seconds or had a sustained response to the $50 \mathrm{~Hz}$ tetanus, one hour following antagonism of muscle relaxants. One patient in Group 1 was intentionally not given neostigmine and glycopyrrolate at the end of surgery. Estimated power for comparisons of clinical duration in the atracurium groups (Groups 1 and 2) is $\geq 0.90$ ( $\alpha$ at 0.05 , two tailed, $\beta$ at $0.10, \sigma=10$ minutes, $\Delta=15$ minutes).

\section{Discussion}

Previous reports studying the interaction between muscle relaxants and aminoglycoside antibiotics have been in vitro or in vivo animal studies. To our knowledge, this is the first prospective study that evaluates the interaction in human subjects.

The time to recovery of a $\mathrm{T} 4 / \mathrm{T} 1$ ratio to 0.70 after the administration of a nondepolarizing muscle relaxant is rarely measured by authors who study the recovery characteristics of the neuromuscular blockade produced by these relaxants. However, we measured that index in the present study because $\mathrm{T} 4 / \mathrm{T} 1$ ratio is presumably an indicator of decreased prejunctional transmission, ${ }^{3}$ and because gentamicin is known for its prejunctional inhibitory effect. ${ }^{4}$

Gentamicin and tobramycin were studied together because both are aminoglycosides and both are known to inhibit neuromuscular transmission. ${ }^{4,6}$ They are also used at the same clinical dosages in man. Currently, gentamicin is believed to prolong the neuromuscular block 
produced by atracurium. ${ }^{7}$ However, this notion is based on a previous study done in cats, in which the results did not support the author's conclusion, as gentamicin, at dosages of 1 and $3 \mathrm{mg} \cdot \mathrm{kg}^{-1}$ did not cause a significant difference in twitch depression between the control measures and those in presence of the antibiotic. The results of the present study suggest that gentamicin and tobramycin, when used at therapeutic serum levels (1 to $\left.10 \mu \mathrm{g} \cdot \mathrm{kg}^{-1}\right)$, do not potentiate or prolong the action of atracurium in man. However, the same antibiotics, used at the same dosage, significantly prolong the clinical duration of vecuronium and the time to spontaneous recovery of a $\mathrm{T} 4 / \mathrm{T} 1$ ratio of 0.70 .

No animal studies have compared the relative effect of gentamicin and tobramycin interacting with muscle relaxants. However, the results of investigation done in animal preparations, in the absence of muscle relaxant, suggest that gentamicin may be a more potent inhibitor of neuromuscular transmission than tobramycin. ${ }^{5}$ Those results were obtained with extremely high drug doses and it was found that the maximal inhibitory effect with tobramycin was obtained with a dose of $60-100 \mathrm{mg} \cdot \mathrm{kg}^{-1}$ whereas the comparable maximal effect was produced by $50 \mathrm{mg} \cdot \mathrm{kg}^{-1}$ of gentamicin. In the present human study, both tobramycin and gentamicin given at therapeutic doses showed a similar degree of inhibition of neuromuscular transmission in the presence of muscle relaxants. In fact, while neither influenced the atracurium block, they both increased the clinical duration and the time to spontaneous recovery of $\mathrm{T} 4 / \mathrm{Tl}$ ratio of 0.70 of the neuromuscular blockade produced by vecuronium. We cannot explain why gentamicin and tobramycin do not affect the atracurium block while they affect the vecuronium block. We can only speculate that if the effect of those antibiotics was additive to the effect of the muscle relaxants, then a similar increase in the clinical duration and time to spontaneous recovery of a T4/T1 ratio of 0.70 would be expected for both atracurium and vecuronium. In fact, both muscle relaxants have similar spontaneous recovery characteristics. ${ }^{9}$ Therefore the interaction between vecuronium and those antibiotics could be one of synergism which may have increased the binding of vecuronium to neuromuscular receptors. Another possible explanation for the difference between vecuronium and atracurium in presence of these antibiotics may be the different pharmacokinetics of these two muscle relaxants: ${ }^{10,11}$ atracurium has a shorter half-life than vecuronium and it is possible that the serum concentrations of atracurium after a single bolus decreased rapidly, preventing interaction with the antibiotics.

The accepted therapeutic serum concentrations of gentamicin and tobramycin in humans vary from 1 to 10 $\mu \mathrm{g} \cdot \mathrm{ml}^{-1}$, so that 15 minutes after administration of an appropriate dose of antibiotics, the senum levels should not be higher than $10 \mu \mathrm{g} \cdot \mathrm{ml}^{-1}$, and not lower than $1 \mu \mathrm{g} \cdot \mathrm{ml}^{-1}$ prior to the administration of the next dose. In the present study, the variation in serum levels of those antibiotics between 1 and $10 \mu \mathrm{g} \cdot \mathrm{ml}^{-1}$ had no relation to the clinical duration or the time to spontaneous recovery of $\mathrm{T} 4 / \mathrm{T} 1$ ratio of 0.70 of the neuromuscular block produced by the studied muscle relaxants. This means that lower serum levels of these antibiotics prolonged the vecuronium block as much as the higher levels. In the case of the atracurium block, it means that the neuromuscular blockade was not prolonged even for the patients who received an appropriate dose of gentamicin or tobramycin immediately before or during surgery. It is, however, not excluded that toxic levels of those antibiotics could prolong the atracurium block.

Reversal of the neuromuscular block was not assessed in this study because our patients did not have the same level of neuromuscular block at the time of reversal with neostigmine. Nevertheless, evaluation of neuromuscular function one hour after the administration of neostigmine represents a good way of detecting neuromuscular block which was difficult to antagonize or which presented a recurrence. In fact, one patient treated with tobramycin and receiving vecuronium was unable to sustain a head lift for five seconds one hour after reversal with neostigmine. That patient had received a single bolus injection of vecuronium $0.1 \mathrm{mg} \cdot \mathrm{kg}^{-1}$ at the induction of anaesthesia. After the spontaneous recovery of a T $4 / \mathrm{T} 1$ ratio of 0.70 which took 106 minutes, the patient was given neostigmine $2.5 \mathrm{mg}$ and glycopyrrolate $0.6 \mathrm{mg}$ and was brought to the recovery room while breathing spontaneously, and the EMG monitoring was discontinued. According to the protocol, the patient was assessed by the recovery room nurse one hour later, and he could not sustain a head lift although he was awake and cooperative. He did not show any respiratory distress, and without additional neostigmine, recovered his strength in the next hour. This case and a previous report of prolonged action of vecuronium in a patient treated with gentamicin ${ }^{12}$ suggest that reversal of neuromuscular blockade produced by vecuronium can be a problem in the presence of these antibiotics.

In conclusion, vecuronium shows a significant increase in its duration of action in the presence of therapeutic serum levels of gentamicin or tobramycin while atracurium does not. Therefore, for patients treated with these antibiotics, the use of atracurium might present an advantage over vecuronium when a prolonged block is not desired. 


\section{References}

1 Sokoll MD, Gergis SD. Antibiotics and neuromuscular function. Anesthesiology 1981; 55: 148-59.

2 Kronenfeld MA, Thomas SJ, Turndorf $H$. Recurrence of neuromuscular blockade after reversal of vecuronium in a patient receiving polymyxin/amikacin sternal irrigation. Anesthesiology 1986; 65: 93-4.

3 Bowman WC. Prejunctional and postjunctional cholinoreceptors at the neuromuscular junction. Anesth Analg 1980; 59: $935-43$.

4 Torda $T$. The nature of gentamicin-induced neuromuscular block. Br J Anaesth 1980; 52:325-9.

5 Welles JS, Emmerson JL, Gibson WR, Nickander R, Owen $N V$, Anderson RC. Preclinical toxicology studies with tobramycin. Toxicol Appl Pharmacol 1973; 25: 398-409.

6 Waterman PM, Smith RB. Tobramycin-curare interaction. Anesth Analg 1977; 56: 587-8.

7 Stoelting $R K$. Pharmacology and physiology in anesthetic practice. Philadelphia: J.B. Lippincott, 1987, p. 205.

8 Chapple DJ, Clark JS, Hughes $R$. Interaction between atracurium and drugs used in anaesthesia. Br J Anaesth 1983; 55: 17S-22S.

9 Pearce AC, Casson WR, Jones RM. Factors affecting train-of-four fade. Br J Anaesth 1985; 57: 602-6.

10 Ward S. Neill EAM, Weatheriey BC, Corall IM. Pharmacokinetics of atracurium besylate in healthy patients (after a single I.V. bolus dose). Br J Anaesth 1983; 55: 113-8.

11 Cronnelly $R$, Fisher DM, Miller RD, Gencarelli $P$, Nguyen-Gruenka L, Castognoli N. Pharmacokinetics and pharmacodynamics of vecuronium (Org NC45) and pancuronium in anesthetized humans. Anesthesiology 1983; 58: 405-8.

12 Shanks AB, Long T, Aitkenhead AR. Prolonged neuromuscular blockade following vecuronium. Br J Anaesth 1985; 57: 807-10.

\section{Résumé}

L'interaction entre les aminoglycosides (tobramycinegentamicine) et le vécuronium ainsi que l'atracurium a été étudiée de façon prospective chez 44 patients dont 22 avaient des niveaux sanguins therapeutiques de tobramycine ou de gentamicine et 22 constituaient des sujets témoins qui ne prenaient pas d'antibiotiques. Le début d'action, la durée clinique ainsi que le temps nécessaire pour une récupération du rapport T4/TI à 0.70 ont été mesurés. Aucune différence statistiquement significative n'a pu être démontrée en ce qui a trait au début d'action des myorelaxants, mais les patients recevant de la gentamicine ou de la tobramycine d qui l'on a administré du vécuronium ont présenté des temps de récupération significativement plus longs que le groupe témoin $(P<0.01$ pour la durée clinique et $P<0.0005$ pour le temps de récupération du rapport $T 4 / T I$ à 0.70 ). L'atracurium n'a pas semblé subir d'influence de la tobramycine ou de la gentamicine utilisés à un niveau thérapeutique. Nous concluons que pour des patients traités avec de la tobramycine ou de la gentamicine l'atracurium peut présenter certains avantages sur le vécuronium quand on veut éviter un bloc neuromusculaire prolongé. 